\title{
IL-22 modulates inflammatory properties of human primary aortic smooth muscle cells
}

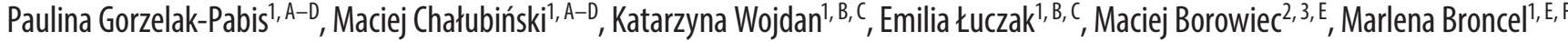 \\ ${ }^{1}$ Laboratory of Tissue Immunopharmacology, Department of Internal Diseases and Clinical Pharmacology, Medical University of Lodz, Poland \\ ${ }^{2}$ Immunopathology and Genetics Laboratory, Department of Pediatrics, Oncology, Hematology and Diabetology, Medical University of Lodz, Poland \\ ${ }^{3}$ Department of Clinical Genetics, Medical University of Lodz, Poland
}

A - research concept and design; $\mathrm{B}$ - collection and/or assembly of data; $\mathrm{C}$ - data analysis and interpretation;

$D$ - writing the article; $E$ - critical revision of the article; $F$ - final approval of article

\section{Address for correspondence}

Paulina Gorzelak-Pabis

E-mail:kluzman.p@gmail.com

\section{Funding sources}

This study was supported by the grant for young investigators No: 502-03/5-165-01/502-54-147 and statutory funds from the Medical University of Lodz №: 503/5-165-01/503-51-002.

Conflict of interest

None declared

Received on November 26, 2015

Revised on February 14, 2016

Accepted on March 16, 2016

\begin{abstract}
Background. IL-22 is expressed at barrier surfaces, which suggests its critical role in the maintenance of normal barrier homeostasis and tissue repair. IL-22 can both promote pathological inflammation and prevent the destruction of tissues. The functional outcomes of IL-22 on vascular smooth muscle cells, which are shown to regulate immune processes within the vascular wall and which are involved in certain pathologies, have not been analyzed.
\end{abstract}

Objectives. The effect of IL-22 on the expression of novel anti- and pro-inflammatory and barrier disrupting cytokines, apoptosis and the expression of adhesive molecules in human primary aortic smooth muscle cells (AOSMC) was investigated.

Material and methods. Human AoSMC were induced with IL-22 for $24 \mathrm{~h}$ in vitro. The influence of IL-22 on IL-35 subunits EBI3 and p35, LL-33, IFN- $\gamma$ and VEGF mRNA expression in Ao-SMC were assessed using real-time PCR. Additionally, the surface expression of ICAM-1 and apoptosis of AoSMC were analyzed in the flow cytometer.

Results. IL-22 caused a 2- and 3-fold increase of mRNA expression of the EBI3 and p35 IL-35 subunits, and a 40\% decrease of IL-33 mRNA expression in A0SMC. Additionally, L-22 decreased ICAM-1 expression on the surface of AOSMC by 30\%. However, L-22 affected neither IFN-y and VEGF mRNA expression in AOSMC nor their apoptosis and viability.

Conclusions. Our data suggest that IL-22, which is released by Th22 and NK cells, may be an agent affecting the inflammatory response of AoSMC, and thus it may regulate immune homeostasis of the vascular wall.

Key words: ICAM-1, IL-22, th22 cells, AoSMC

DOI

10.17219/acem/62218

Copyright

Copyright by Author(s)

This is an article distributed under the terms of the

Creative Commons Attribution Non-Commercial License

(http://creativecommons.org/licenses/by-nc-nd/4.0/) 


\section{Introduction}

Interleukin-22 (IL-22) is a class $2 \alpha$-helical cytokine of the IL-10 family, which consists of IL-10, IL-19, IL-22, IL-24 and IL-26. ${ }^{1}$ It is produced by cells of hematopoietic origin: Both the innate lymphocytes, NK cells, and the adaptive arm of the immune system, such as Th cells termed Th22 cells. ${ }^{2}$ IL-22, which is preferentially released under Th-17-polarised conditions, signals through a class 2 receptor (IL-22R) composed of the subunits IL-22R1 and IL-10R2. ${ }^{3,4}$ The functional IL-22R is thought to be restricted to the nonhematopoietic cells of the skin, intestine, liver, lung and kidney. ${ }^{5}$

IL-22 expressed at barrier surfaces was shown to promote epithelial cell proliferation, survival and repair in the skin, airway and intestine. It induces the production of pro-inflammatory and remodeling cytokines, enhances proliferation and migration of colonic epithelial cells and promotes proliferative and anti-apoptotic pathways. ${ }^{2,6,7}$ This suggests its critical role in the maintenance of normal barrier homeostasis and tissue repair. However, IL-22 is involved in the development of chronic inflammatory conditions, such as psoriasis, inflammatory bowel disease, rheumatoid arthritis or asthma. ${ }^{8-10}$ Very recently, Th22 cells have been found in atherosclerotic plaques, which indicates the possible influence of IL-22 on vascular wall tissues, including vascular smooth muscle cells (VSMC). ${ }^{11,12}$

VSMC, which comprise the medial layer of the artery wall, are one of the tissue factors that regulate physiological processes, contributing to the establishment of local homeostasis. As they serve as a strong source of proinflammatory cytokines and chemokines released to the microenvironment in response to their activation, they may be involved in chronic inflammatory pathologies, i.e. atherosclerosis, where increased apoptosis of VSMC is observed as is the expression of adhesion molecules. ${ }^{13-15}$ Although Th22 cells have been found in the vascular wall, the effect of IL-22 on VSMC has not yet been investigated. Therefore, the aim of the present study was to assess the effect of IL-22 on the inflammatory profile of human primary aortic smooth muscle cells (AoSMC). For this purpose, we investigated the influence of IL-22 on the mRNA expression of novel, anti- and pro-inflammatory cytokines: IL-35 subunits and IL-33, respectively, which both may modulate the local immune environment and whose role in VSMC has not yet been described. Additionally, we analyzed the effect of IL-22 on the mRNA expression of IFN- $\gamma$ and VEGF - well known endothelial barrier disrupting agents, surface expression of adhesive molecule ICAM-1 and apoptosis of AoSMC. ${ }^{16,17}$

\section{Material and methods}

\section{Cells}

Human primary Aortic Smooth Muscle Cells (AoSMC) (Lonza, Clonetics, CC-2571, Basel, Switzerland) were expanded in Smooth Muscle Cell Basal Medium (Lonza, Clonetics, CC-3181, Basel, Switzerland), supplemented with SmGM-2 BulletKit (Lonza, Clonetics, CC-4149, Basel, Switzerland). After covering $80-90 \%$ of the base of a T-flask, the AoSMC were trypsinized with $0.05 \%$ trypsin with $0.02 \%$ EDTA (SAFC Biosciences, 59417C, Kansas, USA) for $4 \mathrm{~min}$ and neutralized using Trypsin

Fig. 1. The effect of $\mathrm{IL}-22(1 \mathrm{ng} / \mathrm{mL})$ on $\mathrm{mRNA}$ expression of $\mathrm{A})$ anti- and pro-inflammatory cytokines and $\mathrm{B})$ barrier disrupting agents in AoSMC (24 h) assessed by real-time $p c r ;\left(n=6\right.$, from 3 independent experiments), (mean \pm SEM); unstim.- unstimulated control, ${ }^{*} p<0.05$, ${ }^{* *} p<0.01$ : statistically significant as compared to unstimulated control; ns - statistically not significant in comparison to unstimulated control
A
B
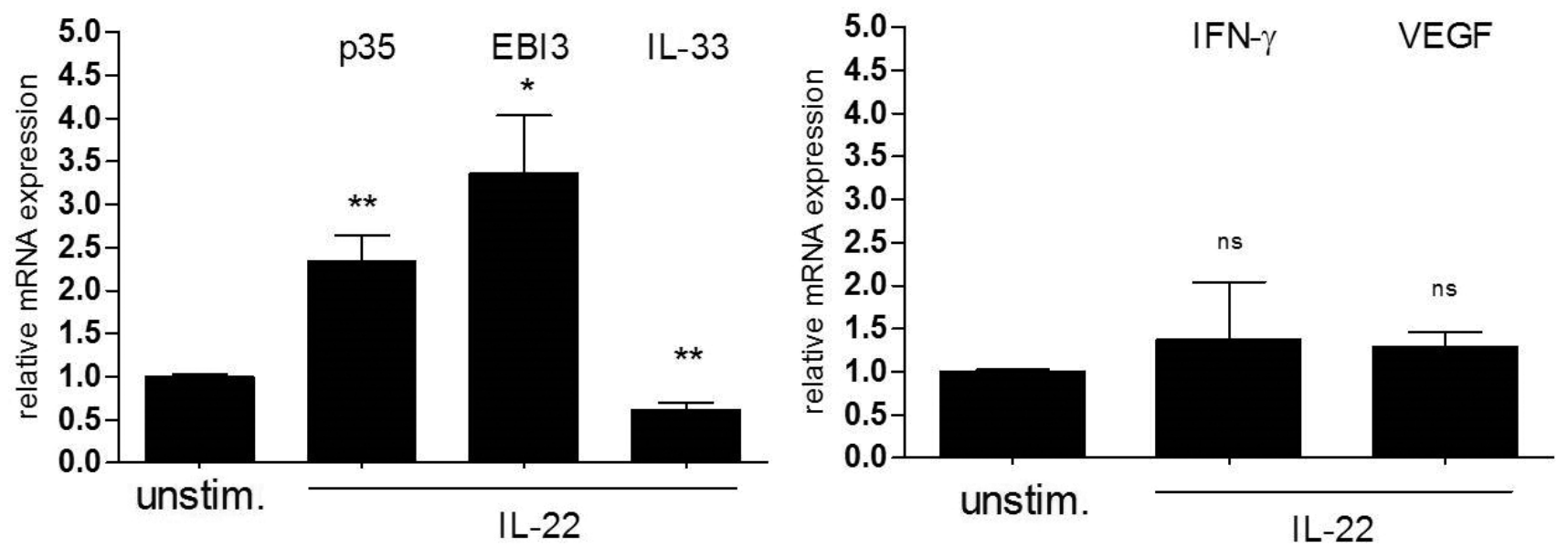
Neutralizing Solution (Lonza, Clonetics, CC-5002, Basel, Switzerland) for further experiments.

\section{Cell culture}

AoSMC were seeded on 12-well plates at a density of 50,000 cells per well in proper media. After reaching 80-90\% confluence, AoSMC were induced with IL-22 (1 ng/mL) (Biolegend, 571302, San Diego, USA) for $24 \mathrm{~h}$ in order to measure the expression of particular cytokine mRNAs by real-time PCR as well as analyze their apoptosis, necrosis and viability, and ICAM-1 (CD54) surface expression by flow cytometry. The optimal concentration of IL-22 used in this study was determined in a set of pilot experiments.

\section{Real time PCR}

mRNA was isolated using the RNeasy Mini Kit (Qiagen, Hilden, Germany) kit, following the manufacturer's instructions. Potential genomic DNA contamination was removed with on-column DNase I digestion and $10 \mu \mathrm{g}$ of mRNA was reverse transcribed with a High-Capacity cDNA Kit (Applied Biosystems, Foster City, USA). PCR was then carried out using an Applied Biosystems 9700HT Fast Real-Time PCR System (Applied Biosystems). The PCR mixture consisted of cDNA solution, SYBR-Green PCR Mastermix (Applied Biosystems) and both sense and antisense primers. The reaction was conducted as follows: $4 \mathrm{~min}$ at $95^{\circ} \mathrm{C}$, followed by 40 cycles of $15 \mathrm{~s}$ at $95^{\circ} \mathrm{C}$ and $60 \mathrm{~s}$ at $60^{\circ} \mathrm{C}$. EF1- $\alpha$ was amplified as a house-keeping gene. p35, EBI3, IL-33, IFN- $\gamma$ and VEGF mRNA expression was normalized to EF1- $\alpha$ using $\Delta \Delta C t$ calculation. Primers used for (1) p35 forward: TTC ACC ACT CCC AAA ACC TGC, reverse: GAG GCC AGG CAA CTC CCA TTA G; (2) EBI3 forward: CAG CTT CGT GCC TTT CAT AA, reverse: CTC CAA CTG CAC CTG TAG C; (3) IL-33 forward: TGA GTC TCA ACA CCC CTC AAA TG, reverse: GGC ATG CAA CCA GAA GTC TTT T; (4) IFN- $\gamma$ forward: TCT CGG AAA CGA TGA AAT ATA CAA GTT AT reverse: GTA ACA GCC AAG AGA ACC CAA AA; (5) VEGF were forward: GAT TGA GAC CCT GGT GGA CAT C, reverse: CAC ACA GGA GGG CTT GAA GA. Those used for EF-1 $\alpha$ were forward: CTG AAC CAT CCA GGC CAA AT, reverse: GCC GTG TGG CAA TCC AAT.

\section{Apoptosis and ICAM-1 surface expression in AoSMC assessment in flow cytometry}

AoSMC were trypsinized after $24 \mathrm{~h}$ of induction with IL-22 (1 ng/mL). Apoptosis was assessed in a BeckmanCoulter FC500 flow cytometer using Annexin V-FITC and propidium iodide (PI) co-staining (FITC-Annexin V Apoptosis Detection Kit, BD Pharmingen, 556547, San Diego, USA). Annexin-V(-) and PI(-) cells were considered as living cells, Annexin- $\mathrm{V}(+)$ and $\mathrm{PI}(-)$ as early-apoptotic cells, Annexin-V(+) and $\mathrm{PI}(+)$ as late-apoptotic cells, and Annexin-V(-) and $\mathrm{PI}(+)$ as necrotic cells.

In order to assess ICAM-1 surface expression on AoSMC, PE anti-human CD54 antibodies (BioLegend, 322708) were used. For analysis, the percentage changes of Mean Fluorescence Intensity (MFI) of were used.

\section{Statistical analysis}

The results are presented as mean \pm SEM for variables with a normal distribution of values. The distribution of particular variables was verified by the Shapiro-Wilk W test, whereas the Levene test was performed to test homogeneity of variances. In the case of normal distribution and homogenous variance, the significance of differences between 2 groups was estimated using the Student $\mathrm{t}$ test for independent trials. However, if any of these criteria were not fulfilled, a Mann-Whitney U test was used for analysis of the differences between the 2 groups. A p-value $<0.05$ was considered to be statistically significant. All analyses were conducted with STATISTICA v. 12.5 software (StatSoft, Inc., Tulsa, USA).

\section{Results}

The effect of IL-22 on anti- and pro-inflammatory cytokines: IL-35 subunits p35 and EBI3, IL-33 mRNA expression in AoSMC

The effect of IL-22 on the mRNA expression of antiand pro-inflammatory cytokines in AoSMC was first investigated. EBI3 and p35 IL-35 subunits, IL-33 and IFN- $\gamma$ mRNA expression in AoSMC was assessed in real-time PCR after $24 \mathrm{~h}$ of culture (Fig. 1A). In AoSMC induced with IL-22, an almost 2.5-fold increase of IL-35 p35 subunit mRNA expression was observed as compared to the unstimulated control ( $\mathrm{p}<0.01$ ). Similarly, IL-35 EBI3 subunit mRNA expression was more than 3 times higher than that of unstimulated AoSMC upon IL-22 induction ( $\mathrm{p}<0.05$ ). On the other hand, IL-33 mRNA expression was seen to halve upon stimulation of AoSMC with IL-22 as compared to the control ( $\mathrm{p}<0.01)$.

The effect of IL-22 on barrier disrupting cytokines: IFN- $\gamma$ and VEGF mRNA expression in AoSMC

Next, we assessed the effect of IL-22 on the mRNA expression of cytokines involved in the regulation of barrier functions of tissues such as endothelium (Fig. 1B). For this purpose, IFN- $\gamma$ and VEGF mRNA expression was analyzed after $24 \mathrm{~h}$ of culture of AoSMC with IL-22 (1 ng/mL). IL-22 did not change IFN- $\gamma$ mRNA expression in AoSMC, as compared to the unstimulated cells 
( $p$ > 0.05). Similarly, IL-22 did not significantly change VEGF mRNA expression in AoSMC, as compared to the unstimulated cells $(\mathrm{p}>0.05)$.

\section{The effect of IL-22 on AoSMC ICAM-1 (intercellular} adhesion molecule-1) expression

For further analysis of the effect of IL-22 on the inflammatory profile of AoSMC, the surface expression of ICAM-1 on AoSMC was assessed in a flow cytometer after $24 \mathrm{~h}$ of culture, based on the percentage changes of Mean Fluorescence Intensity (MFI). Upon induction of AoSMC by IL-22 (1 ng/mL), a significant decrease of surface ICAM-1 expression was observed (mean: down to $69.2 \pm 7.2 \% ; \mathrm{p}<0.05)$ as compared to unstimulated cells (Fig. 2A, B).

\section{The effect IL-22 on AoSMC apoptosis and viability}

In the next step, the effect of IL-22 on AoSMC survival was analyzed. The apoptosis and viability of AoSMC were assessed after $24 \mathrm{~h}$ of culture by flow cytometry (Fig. 3). IL-22 did not affect AoSMC apoptosis or necrosis, as the percentages of $(\mathrm{Ax}+\mathrm{Pi}-$ plus $\mathrm{Ax}+\mathrm{Pi}+)$ or $\mathrm{Ax}-\mathrm{Pi}+$ cells, upon induction with IL-22, were found to be comparable to those of unstimulated AoSMC (mean $[\mathrm{Ax}+\mathrm{Pi}$ - plus $\mathrm{Ax}+\mathrm{Pi}+$ ] cells: $30.0 \pm 16.2 \%$ versus $26.5 \pm 12.9 \%$, respectively, $\mathrm{p}>0.05$ ) (Fig. 3A); (mean Ax-Pi+ cells: $2.5 \pm 1.1 \%$ versus $2.9 \pm 1.4 \%$, respectively, $\mathrm{p}>0.05$ ) (Fig. $3 \mathrm{~B}$ ). Similarly, IL-22 was not observed to have any effect on AoSMC viability: IL-22 did not change the percentages of Ax-Picells, with mean values of $67.3 \pm 14.9 \%$ respectively versus $70.6 .8 \pm 12.4 \%$ in controls ( $p>0.05$ ) (Fig. 3C).

\section{Discussion}

The study analyzes the influence of IL-22 on the inflammatory profile of VSMC. It can be seen that IL-22, which may be released by Th22 cells or NK cells penetrating the vascular wall, may affect the mRNA expression of certain cytokines and surface expression of adhesion molecules on VSMC.

As the biological effect of IL-22 on VSMC is unexplored, it was decided to investigate the influence of IL-22 on the mRNA expression of novel anti- and proinflammatory cytokines in human AoSMC. Firstly, it was observed that IL-22 induced mRNA expression of EBI3 (Epstein-Barr virus-induced gene-3) and p35 - subunits of anti-inflammatory IL-35, a novel IL-12 family cytokine, relevant for coronary artery disease. ${ }^{18,19} \mathrm{IL}-35$, whose EBI3 subunit is expressed in aortic smooth muscle cells, was shown to inhibit both Th1- and Th2-mediated chronic inflammatory conditions. ${ }^{20,21}$

On the other hand, we have shown that IL-22 decreased mRNA expression of IL-33, a pro-inflammatory tissue-derived danger signal relevant in certain chronic inflammatory diseases, by roughly $40 \%$ in AoSMC. ${ }^{22-24}$ This suggests that IL-22 may diminish VSMC-derived IL-33-driven inflammatory processes in the vascular wall, such as survival and cytokine production by immune cells and the generation of Th-2 type immune responses. ${ }^{25-26}$ Thus, through the effect on IL-35 and IL-33 expression in AoSMC, IL-22 might affect VSMC to reduce local inflammatory processes within the vascular wall.

The endothelial barrier plays a key role in inflammatory processes. Both IFN- $\gamma$ and VEGF are well know agents decreasing endothelial barrier functions. ${ }^{17}$ As VSMC are

Fig. 2. The effect of IL-22 on ICAM-1 expression on AoSMC. A) The effect of IL-22 (1 ng/mL) on the percentage change of surface ICAM-1 expression on AOSMC (24 h) by flow cytometry; ( $n=4$, from 4 independent experiments), (mean \pm SEM); ${ }^{*} p<0.05$; (MFI - mean fluorescence intensity); B) Representative overlays of IL-22-induced changes of ICAM-1 surface expression on AOSMC, unstim. - unstimulated control; flow cytometry

A

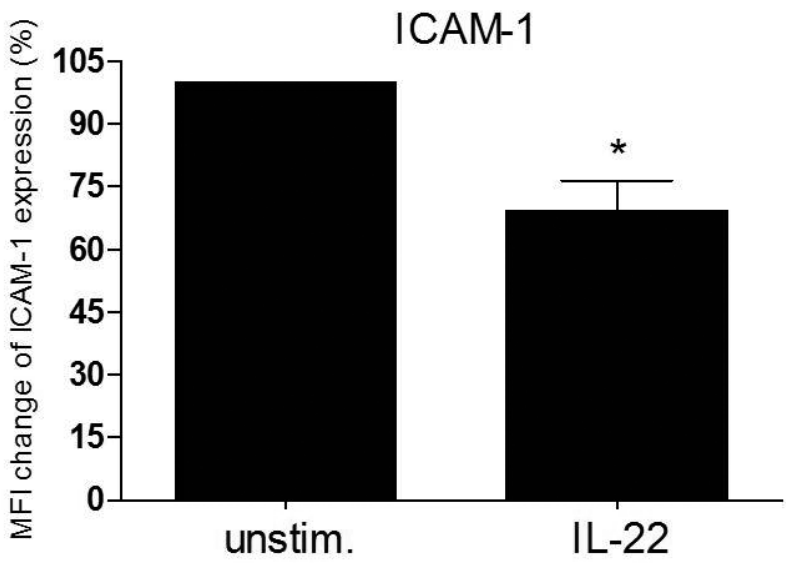

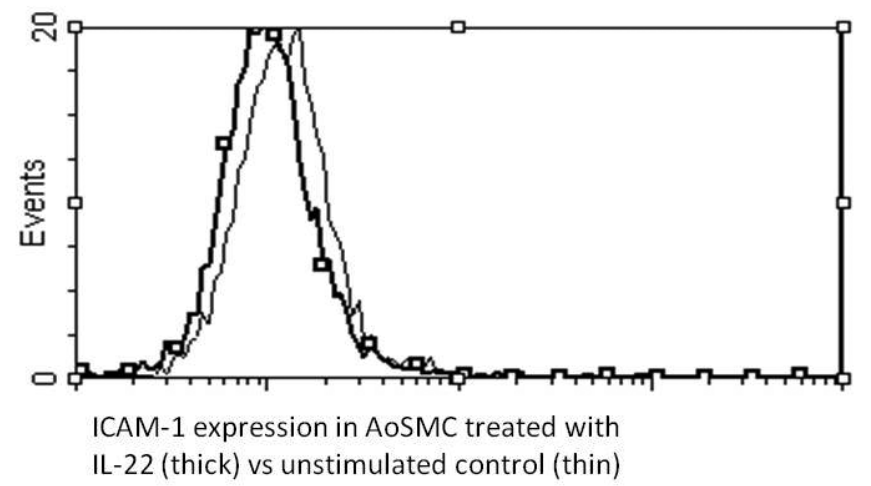


Fig. 3. The effect of IL-22 on AoSMC death and viability assessed by flow cytometry (24 h). The effect of IL-35 on A) apoptotic, B) necrotic and C) viable AoSMC percentages (24 h) ( $n=3$, from 3 independent experiments), (mean \pm SEM); (Annexin V(+) - Annexin V-positive AoSMC, PI(+) - Propidium iodide-positive AoSMC); (D) Representative dot blots of unstimulated (unstim.), IL-22 (1 ng/mL)-induced AoSMC; x axis - Annexin V-FITC, y axix - propidium iodide, flow cytometry

A

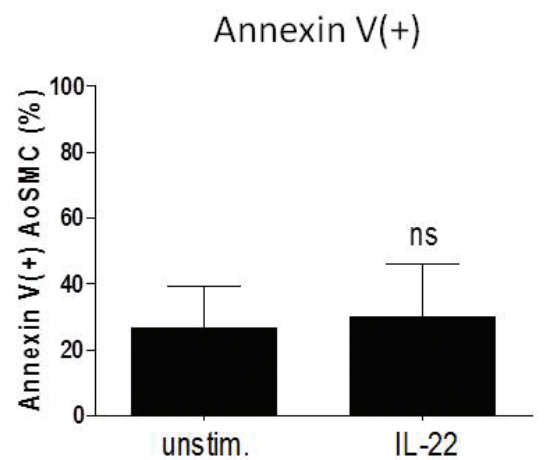

B

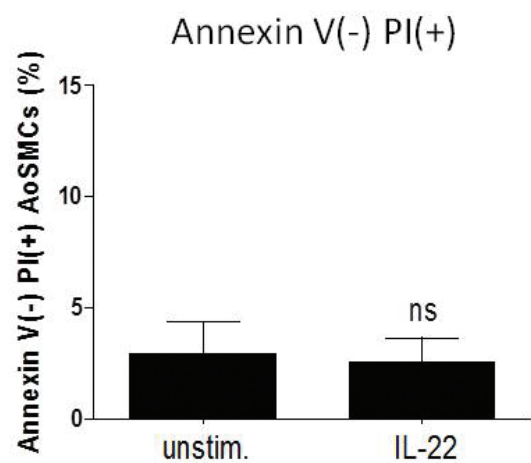

C

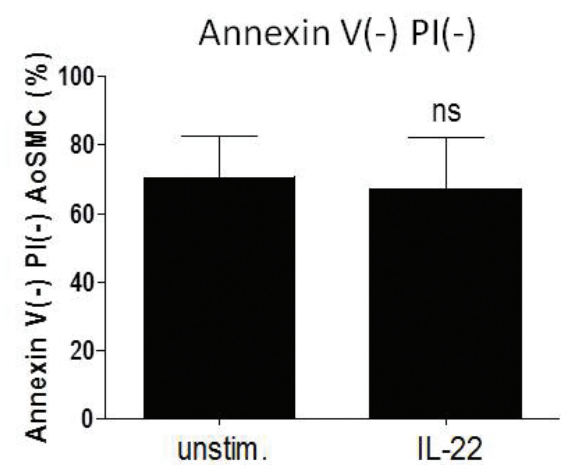

D

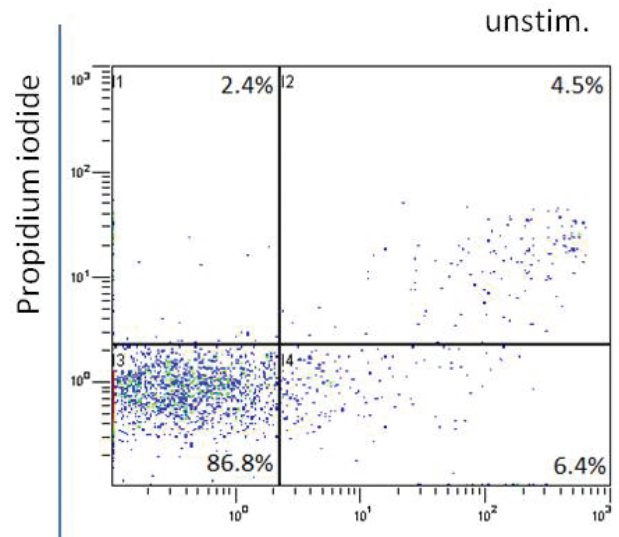

IL-22

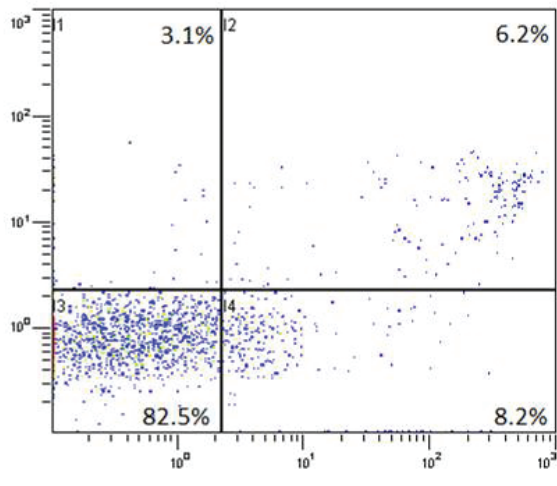

Annexin V-FITC

located in the endothelial layer neighborhood, they might affect its barrier functions via cytokine release to the microenvironment. The question that we raised concerned whether IL-22 may influence the mRNA expression of cytokines, decreasing barrier functions. However, according to our results IL-22 affected neither IFN- $\gamma$ nor VEGF mRNA expression, in AoSMC.

Leukocyte-tissue interactions require the expression of ICAM-1, which may serve as an adhesion molecule for leukocytes and facilitate cell infiltration. ${ }^{27}$ We observed that AoSMC weakly express ICAM-1. Nevertheless, ICAM-1 expression on AoSMC decreased by approximately 30\% upon stimulation with IL-22, which suggests that IL-22 might slightly decrease the accessibility of arterial wall tissues for immune cells in contrast to other typical inflammatory cytokines, such as TNF- $\alpha$, IFN- $\gamma$ and IL-1 $\beta$, and affect VSMC migration. ${ }^{15,28,29}$
Recent results describe the effect of IL-22 on apoptosis of the cells of certain tissues. Although IL-22 was seen, firstly, to prevent apoptosis of hepatic stellate cells, secondly, to induce the expression of antiapoptotic B-cell lymphoma cells and, finally, to demonstrate an ability to promote the survival of airway SMC, the effect of IL-22 on apoptosis of AoSMC has not been investigated. ${ }^{10,30}$ However, IL-22 was not observed to have any effect on apoptosis, necrosis or survival of resting AoSMC in normal conditions in our study. This data indicates that IL-22 does not affect apoptosis of VSMC.

To sum up, the results of our study showing certain effects of IL-22 on IL-35 subunits, IL-33 and ICAM-1 expression in AoSMC, support the assumption that IL-22, which may be released by Th22 and NK cells, may be an agent affecting vascular smooth muscle cell-orchestrated inflammatory processes, and thus may regulate the immune homeostasis of the vascular wall. 


\section{References}

1. Pestka S, Krause CD, Sarkar D, Walter MR, Shi Y, Fisher PB. Interleukin-10 and related cytokines and receptors. Annu Rev Immunol. 2004;22:929-979.

2. Zenewicz LA, Flavell RA. Recent advances in IL-22 biology. Int Immunol. 2011:23:159-163.

3. Kumar P, Rajasekaran K, Palmer JM, Thakar MS, Malarkannan S. IL-22: An evolutionary missing-link authenticating the role of the immune system in tissue regeneration. J Cancer. 2013;4:57-65.

4. Kotenko SV, Izotova LS, Mirochnitchenko OV, et al. Identification of the functional interleukin-22 (IL-22) receptor complex: The IL-10R2 chain (IL-10Rbeta) is a common chain of both the IL-10 and IL-22 (IL-10-related T cell-derived inducible factor, IL-TIF) receptor complexes. J Biol Chem. 2001;276:2725-2732.

5. Sonnenberg GF, Fouser LA, Artis D. Border patrol: Regulation of immunity, inflammation and tissue homeostasis at barrier surfaces by IL-22. Nat Immunol. 2011;12:383-390.

6. Andoh A, Zhang Z, Inatomi O, et al. Interleukin-22, a member of the IL-10 subfamily, induces inflammatory responses in colonic subepithelial myofibroblasts. Gastroenterology. 2005;129:969-984.

7. Pickert G, Neufert C, Leppkes $M$, et al. STAT3 links IL-22 signaling in intestinal epithelial cells to mucosal wound healing. J Exp Med. 2009;206:1465-1472.

8. Ikeuchi H, Kuroiwa T, Hiramatsu N, et al. Expression of interleukin-22 in rheumatoid arthritis: Potential role as a proinflammatory cytokine. Arthritis Rheum. 2005;52:1037-1046.

9. Boniface K, Bernard FX, Garcia M, Gurney AL, Lecron JC, Morel F. IL-22 inhibits epidermal differentiation and induces proinflammatory gene expression and migration of human keratinocytes. J Immunol. 2005;174:3695-3702.

10. Chang Y, Al-Alwan L, Risse PA, et al. Th17-associated cytokines promote human airway smooth muscle cell proliferation. Faseb $\mathrm{J}$. 2012;26:5152-5160.

11. Oliveira RT, Silva RM, Teo FH, et al. Detection of TCD4+ subsets in human carotid atheroma. Cytokine. 2013;62:131-140.

12. Xia Q, Xiang X, Patel S, Puranik R, Xie Q, Bao S. Characterisation of IL-22 and interferon-gamma-inducible chemokines in human carotid plaque. Int J Cardiol. 2014;154:187-189.

13. Tedgui A, Mallat Z. Cytokines in atherosclerosis: Pathogenic and regulatory pathways. Physiol Rev. 2006;86:515-581.

14. Hansson GK, Hermansson A: The immune system in atherosclerosis. Nat Immunol. 2011;12:204-212.
15. Braun M, Pietsch P, Schror K, Baumann G, Felix SB. Cellular adhesion molecules on vascular smooth muscle cells. Cardiovasc Res. 1999;41:395-401.

16. El-Remessy AB, Franklin T, Ghaley N, et al. Diabetes-induced superoxide anion and breakdown of the blood-retinal barrier: Role of the VEGF/UPAR pathway. PLoS One. 2013;8:e71868.

17. Cromer WE, Zawieja SD, Tharakan B, Childs EW, Newell MK, Zawieja DC. The effects of inflammatory cytokines on lymphatic endothelial barrier function. Angiogenesis. 2014;17:395-406.

18. Collison LW, Workman CJ, Kuo TT, et al. The inhibitory cytokine IL-35 contributes to regulatory T-cell function. Nature. 2007;450:566-569.

19. Lin $Y$, Huang Y, Lu Z, et al. Decreased plasma IL-35 levels are related to the left ventricular ejection fraction in coronary artery diseases. PLoS One. 2012;7:e52490.

20. Kempe S, Heinz P, Kokai E, Devergne O, Marx N, Wirth T. Epsteinbarr virus-induced gene-3 is expressed in human atheroma plaques. Am J Pathol. 2009;175:440-447.

21. Wirtz S, Billmeier U, McHedlidze T, Blumberg RS, Neurath MF. Interleukin-35 mediates mucosal immune responses that protect against T-cell-dependent colitis. Gastroenterology. 2011;141:1875-1886.

22. Lamkanfi M, Dixit VM. IL-33 raises alarm. Immunity. 2009;31:5-7.

23. Liew FY, Pitman NI, Mclnnes IB. Disease-associated functions of IL-33: The new kid in the IL-1 family. Nat Rev Immunol. 2010;10:103-110.

24. Miller AM, Xu D, Asquith DL, et al. IL-33 reduces the development of atherosclerosis. J Exp Med. 2008;205:339-346.

25. likura M, Suto H, Kajiwara N, et al. IL-33 can promote survival, adhesion and cytokine production in human mast cells. Lab Invest. 2007;87:971-978.

26. Demyanets S, Konya V, Kastl SP, et al. Interleukin-33 induces expression of adhesion molecules and inflammatory activation in human endothelial cells and in human atherosclerotic plaques. Arterioscler Thromb Vasc Biol. 2011;31:2080-2089.

27. Yotsui $\mathrm{T}$, Yasuda $\mathrm{O}$, Kawamoto $\mathrm{H}$, et al. Aspirin prevents adhesionof $\mathrm{T}$ lymphoblasts to vascular smooth muscle cells. FEBS Lett. 2007;581:427-432.

28. Entwistle J, Hall CL, Turley EA. HA receptors: Regulators of signalling to the cytoskeleton. J Cell Biochem. 1996;61:569-577.

29. Maier KG, Sadowitz B, Cullen S, Han X, Gahtan V. Thrombospondin1 -induced vascular smooth muscle cell migration is dependent on the hyaluronic acid receptor CD44. Am J Surg. 2009;198:664-669.

30. Kong $X$, Feng $D$, Wang $H$, et al. Interleukin-22 induces hepatic stellate cell senescence and restricts liver fibrosis in mice. Hepatology. 2012;56:1150-1159. 